\title{
A Prospective Randomized Study Comparing Total Laparoscopic Hysterectomy (TLH) Versus Minilaparotomy Hysterectomy (MLH) for benign Uterine Disorders in a Low-Resource Setting: Original Peri-Operative Outcome
} Article

\author{
Mahmoud Mohamed Awad', Adel Fathi Mohamed ${ }^{2}$, Hossam Elsayed Gouda', \\ Yousef Omar Aboelkhir ${ }^{1}$, Tarek Shokeir ${ }^{1}$ and Mohamed Sayed Abdelhafez ${ }^{1}$
}

${ }^{1}$ Department of Obstetrics and Gynecology, ${ }^{2}$ Department of Oncology Center,

Mansoura University Hospital, Faculty of Medicine, Mansoura, Egypt

\begin{abstract}
Aim: To compare the perioperative outcomes between total laparoscopic hysterectomy (TLH) and minilaparotomy hysterectomy (MLH) in patients with benign uterine lesions.

Study design: Randomized controlled trial.

Patients and Methods: This study was conducted on 80 women suffering from benign uterine lesions and prepared for total abdominal hysterectomy in a tertiary university hospital in a low-resource setting. Patients were randomized one day before surgery into two groups; group $1(\mathrm{TLH} ; \mathrm{n}=40)$ and group $2(\mathrm{MLH} ; \mathrm{n}=40)$. The primary outcome measure was the estimated amount of blood loss. The secondary outcome measures were the operative time, changes in the hemoglobin ( $\mathrm{Hb})$ level and hematocrit (Hct) value, hospital stay peroid, intraoperative complications, and related early postoperative complications.

Resuls: MLH has significantly lower amount of estimated blood loss $(52.31 \pm 20.19$ vs $92.11 \pm 26.40 \mathrm{ml} ; P<0.001)$, and significantly lower operative time $(65.26 \pm 8.35$ vs $93.68 \pm 15.58$ minutes; $P<0.001)$, and significantly lower percentage of postoperative drop in $\mathrm{Hb}$ level $(5.32 \pm 1.54 \%$ vs $7.18 \pm 2.94 \% ; P=0.001)$ but with significantly higher hospital stay period $(2.18 \pm 0.39$ vs $1.43 \pm 0.56$ days; $P<0.001)$. Both groups were comparable in the changes in the Hct value and complications rate.

Conclusions: Compared with TLH, MLH offers favorable outcomes in terms of less operative time, less intraoperative bleeding, and short learning curve. Its safety, simplicity, and lower cost probably give the technique a higher privilege in communities with low-resource settings.
\end{abstract}

Key Words: Benign uterine disorders, blood loss, hysterectomy, laparoscopy, minilaparotomy.

Received: 03 April 2021, Accepted: 12 August 2021

Corresponding Author: Mahmoud Mohamed Awad, Department of Obstetrics and Gynecology, Mansoura University Hospitals, Egypt, Tel.: +20 1000337324, E-mail: mahmoudmm8506@gmail.com

ISSN: 2090-7265, November 2021, Vol.11, No. 4

\section{INTRODUCTION}

Hysterectomy is the second most frequently performed gynecologic procedure worldwide after cesarean section (CS). There are many approaches for hysterectomy. These include vaginal hysterectomy $(\mathrm{VH})$, laparoscopic assisted vaginal hysterectomy (LAVH), laparoscopic supracervical hysterectomy (LSH), total laparoscopic hysterectomy (TLH), conventional abdominal hysterectomy and recently minilaparotomy hysterectomy (MLH). The choice of the approach depends on the indication for surgery, the experience of the surgeon, the nature of the disease, the patient characteristics, costs and the patient choice ${ }^{[1]}$.

The rates of hysterectomy by approach were different among countries ${ }^{[2-5]}$. Position statement, systematic reviews and committee opinions support the $\mathrm{VH}$ as the first preferred approach for benign uterine lesions and the TLH as preferable alternative to open hysterectomy when $\mathrm{VH}$ is not feasible or indicated ${ }^{[6-8]}$. However, there are several clinical conditions that the gynecologists probably support a non-vaginal approach including large uterus, nulliparity, lack of previous vaginal delivery, and prior abdominal surgery ${ }^{[9]}$.

A new era started in 1984 when Kurt Semm performed the first LAVH and opened the floodgates for role of laparoscopy in hysterectomy ${ }^{[10]}$, and in 1988, Harry Reich performed the first $\mathrm{TLH}^{[11]}$. The introduction of modern new vessel sealer devices (new electrosurgical and ultrasonic instruments) for hemostasis during laparoscopy has increased the safety of the procedure as there is minimal thermal spread less than $2 \mathrm{~mm}^{[12]}$.

While the abdominal hysterectomy is easy to learn, the benefits of laparoscopic hysterectomy includes shorter 
hospital stay, less intraoperative bleeding, early return to normal daily activity, fewer wound infections and better $\operatorname{cosmosis}^{[7]}$. Despite the advantage of minimally invasive surgery compared with the conventional one, it requires a higher level of technical skills and long learning curve. MLH has come up as a middle path coupling cosmosis and early recovery without need for expensive equipment or training ${ }^{[13]}$.

To our knowledge, there are no RCTs comparing the perioperative outcomes between MLH and TLH in patients with benign uterine lesions. The only published studies comparing TLH and MLH were with retrospective study designs $^{[14,15]}$. Furthermore, from economic point of view, the cost of TLH procedures range from $\$ 500-\$ 2,500^{[16]}$ and these methods are known to be expensive and may become 'in most instances' unsuitable for natives of low-economic standards. MLH, on the other hand, is a cheap, feasible operation and has potentially more favorable outcomes in low-resource settings. Therefore, we aimed to compare prospectively the perioperative outcomes between TLH and MLH in patients with benign uterine lesions with respect to estimated blood loss and related early postoperative complications in a low-resource setting.

\section{MATERIALS AND METHODS}

\section{Patient population}

This was a prospective, randomized, open-label, controlled study conducted in the Department of Obstetrics \& Gynecology, Mansoura University Hospital during the period from December 2016 through May 2019. The study protocol was reviewed and approved by the Mansoura Faculty of Medicine Institutional Research Board (Code \# MD/16.12.51). The trial was registered with ClinicalTrials.gov, identifier NCT03251677.

Women suffering from benign uterine lesions and prepared for total abdominal hysterectomy were selected to enroll in our study. Eligible subjects were interviewed, informed about the study and counseled for participation. They were evaluated regarding the inclusion and exclusion criteria. Women with any of the following criteria were excluded from the study: 1) age is $<18$ years; 2) enlarged uterus ( $>12$ weeks of gestation); 3) fixed uterus; 4) suspicious adnexal masses $(>5 \mathrm{~cm})$; or 5$)$ suspected malignancy. A written informed consent was taken from each woman participating in the study.

\section{Allocation and randomization}

The patients who met the inclusion and exclusion criteria were randomized one day before surgery into two groups; group $1(\mathrm{TLH} ; \mathrm{n}=40)$ and group $2(\mathrm{MLH} ; \mathrm{n}=40)$. The randomization was simple, balanced $(1: 1)$ and determined by the patient's identification number kept within closed sealed envelopes. Women with odd identification numbers were selected for TLH and those with even identification numbers for MLH. All women undergoing TLH were counseled that there was a chance of conversion to conventional laparotomy and those undergoing MLH were counseled about probable extension to larger incision.

\section{Interventions (Surgical techniques)}

Women in the TLH group were subjected to the technique described by Einarsson and Suzuki ${ }^{[17]}$ using harmonic scalpel and bipolar forceps, while women in the MLH group were subjected to the technique described by Fanfani et al ${ }^{[18]}$. All the operations were performed by the same surgeons. In both groups, the surgical team followed the standard procedure of hysterectomy. Prophylactic antibiotic, Ceftriaxone 1 gram (Ceftriaxone (C) $1000 \mathrm{mg}$, Sandoz, Novartis, Switzerland) was given 30 minutes before surgery. Clindamycin $600 \mathrm{mg}$ (DALACIN C TM $600 \mathrm{mg}$, Pfizer, USA) was given if there was a history of allergy to cephalosporins.

\section{Postoperative care}

Postoperatively, vital data were recorded every 6 hours. Parentral antibiotic prophylaxis was continued for 24 hours and then shifted to oral antibiotics for 1 week Oral fluid was allowed after 6 hours and normal diet was resumed the next day. Foley's catheter was removed after 12 hours. For non-complicated cases, outpatient follow-up was scheduled on the $5^{\text {th }}$ postoperative day for removing the dressing, on the $14^{\text {th }}$ postoperative day, and up to one month after operation.

\section{Data collection}

For each woman participating in the study, the following peri-operative data were collected:

1. Demographic data: including age, height, weight, body mass index (BMI), menopausal status (premenopausal or postmenopausal), previous pregnancies, previous deliveries, and previous abdominal surgeries.

2. Preoperative hemoglobin $(\mathrm{Hb})$ level and hematocrit (Hct) value (Pre-Hb and Pre-Hct): measured on the day before surgery.

3. Intraoperative data:

a. Amount of blood loss: estimated in millimeters $(\mathrm{ml})$ by a combination of direct measurement and gravimetric method ${ }^{[19]}$. The direct measurement was performed by collecting most of the lost blood into a suction bottle using a suction apparatus. The gravimetric method was used to estimate the amount of blood loss in the surgical towels and gauzes. The dry surgical towels and gauzes were weighed before surgery. 
Intraoperatively, the towels were used to dry up all the blood in the surgical field then these towels were collected into a sterile metallic bowl which was weighed empty before surgery. Soaked towels and gauzes were collected in the bowl and the bowl with the collected soaked towels and gauzes was handled to the circulating nurse to weigh it by a highly accurate digital balance and then calculate the amount of blood loss and then the amount of blood loss in the towels was calculated by the following formula:-

Amount of blood loss in the soaked towels and gauzes (in $\mathrm{ml}$ ) $=$ weight of the collected soaked towels and gauzes contained within the metallic bowl (in gm) - [weight of the empty towel (in gm) + weight of the towels and gauzes before surgery (in gm)]. After that, the collected blood in the suction bottle was added to the total calculated amount of blood loss in the towels and gauzes. Finally, the amount of fluid used for irrigation was extracted from the total amount of blood loss.

b. Operative time: defined as the time in minutes taken for total hysterectomy, starting with induction of anesthesia up to complete removal of the uterus.

c. Additional operative procedures: such as adhesiolysis or salpingo-oophorectomy (unilateral or bilateral).

d. Uterine weight: measured in grams (gm) by a highly sensitive digital scale.

e. Intraoperative complications: blood loss $>$ $500 \mathrm{ml}$ requiring blood transfusion, visceral or vascular injury.

f. Conversion rate: defined as conversion to conventional laparotomy in the TLH or extension to a larger incision in the MLH.

4. Postoperative $\mathrm{Hb}$ level and Hct value (Post-Hb and Post-Hct): measured 24 hours after surgery.

5. Changes in the $\mathrm{Hb}$ level and Hct value $(\Delta \mathrm{Hb}$ and $\Delta \mathrm{Hct}$ ): calculated by the following formulas:

$$
\begin{aligned}
& \Delta \mathrm{Hb}=[(\text { Pre Hb-Post Hb) } / \text { Pre Hb }] \times 100 \\
& \Delta \mathrm{Hct}=[(\text { Pre Hct }- \text { Post Hct }) / \text { Pre Hct }] \times 100
\end{aligned}
$$

6. Hospital stay days.

7. Early Postoperative complications: defined as any unfavorable events within the first 30 days after surgery, such as paralytic ileus, wound infection, vault hematoma, and vault dehiscence.

\section{Outcome measures}

The primary outcome measure was the estimated amount of blood loss. The secondary outcome measures were the operative time, changes in the $\mathrm{Hb}$ level and Hct value, hospital stay peroid, intraoperative complications, and related early postoperative complications.

\section{Sample size calculation and power analysis}

The sample size was calculated using the computer statistical software $\mathrm{G}^{*}$ Power 3.1.9.2 (t test, tow-tailed significance, alpha error probability $=0.05$, power $=80 \%$, allocation ratio for groups $=1$ ). A sample size of at least 74 women (37 per group) was needed to detect difference of at least $130 \mathrm{ml}$ in the mean blood loss. The estimation of the sample size was based on the previously reported mean blood loss of $241 \pm 238 \mathrm{ml}$ with minilaparotomy and $126 \pm$ $140 \mathrm{ml}$ with laparoscopy with intention-to-treat analysis ${ }^{[14]}$.

\section{Statistical analysis}

The statistical analysis was done using the IBM ${ }^{\circledR}$ SPSS ${ }^{\circledR}$ Statistics, version 22.0 for Windows. Qualitative data were described using frequency and percentage. Quantitative data were described using mean \pm standard deviation and median (minimum and maximum). Chisquare test was used for categorical variables, to compare between different groups with Fischer's exact test as a correction for Chi-Square test when more than $25 \%$ of cells have count less than 5. The Shapiro-Wilk and KolmogorovSmirnov tests were used to test the normality distribution of the continuous variables. The t-test was used to compare the differences among the normally distributed continuous variables while the differences among continuous variables without normal distribution were compared with the MannWhitney U-test. Significance of the obtained results was judged at the $5 \%$ level $(P$ value $<0.5)$.

\section{RESULTS}

As shown in the study flow diagram (Figure 1), 92 women prepared for total abdominal hysterectomy for benign uterine lesions were assessed for eligibility to participate in the study; 12 of them were excluded [not meeting inclusion criteria $(n=9)$, declined to participate $(n=1)$, and other reasons $(n=2)]$. Of the 80 women who were randomized, 2 women in the TLH group discontinued the intervention due to conversion to conventional laparotomy and 1 woman in the MLH group discontinued due to extension to larger incision. Therefore, data of 38 women in the TLH group and 39 patients in the MLH group were subjected to final analysis.

Both groups were comparable in terms of demographic data (Table 1). Regarding the operative data among cases continued the intervention in both groups (Table 2), we found that the mean amount of estimated blood loss was significantly lower in the MLH group than in the TLH arm 
$(52.31 \pm 20.19$ vs $92.11 \pm 26.40 \mathrm{ml} ; \mathrm{P}<0.001)$. Also, the mean operative time was significantly lower in the MLH group than in the TLH group $(65.26 \pm 8.35$ vs $93.68 \pm 15.58$ minutes; $\mathrm{P}<0.001)$. No significant difference between both groups in the mean uterine weight or additional operative procedures including adhesiolysis and salpingooophorectomy.

A noteworthy observation was that global changes in the pre- and post-operative $\mathrm{Hb}$ level and Hct values were comparable in both groups (Table 3). Although there was no difference between both groups in the percentage of postoperative drop in Hct value $(\mathrm{P}=0.572)$, the percentage of postoperative drop in $\mathrm{Hb}$ level was significantly higher in the TLH group than in the MLH arm $(7.18 \pm 2.94 \%$ vs $5.32 \pm 1.54 \% ; \mathrm{P}=0.001)$.

Both intraoperative and postoperative complications rates were comparable in both groups (Table 4). In the TLH group, intraoperative bleeding requiring blood transfusion occurred in 1 case $(2.5 \%)$ and bladder injury (during bladder dissection from the uterus) occurred in another one $(2.5 \%)$. In both cases, conversion to conventional laparotomy was performed. In the MLH group, extension from minilaparotomy incision to the classic Pfannenstiel incision occurred in 1 case $(2.5 \%)$ due to extensive adhesions in the Douglas pouch and absence of good surgical exposure. Paralytic ileus was reported in 1 case $(2.5 \%)$ in the TLH group and wound seroma occurred in 2 cases $(5 \%)$ in the MLH group vs none in the TLH arm (Table 4). Wound infections were managed by antibiotics and dressing and did not need secondary sutures. In non complicated cases, the mean hospital stay period was significantly lower in the TLH group than in the MLH group ( $1.43 \pm 0.56$ vs $2.18 \pm 0.39$ days; $P<0.001)$.

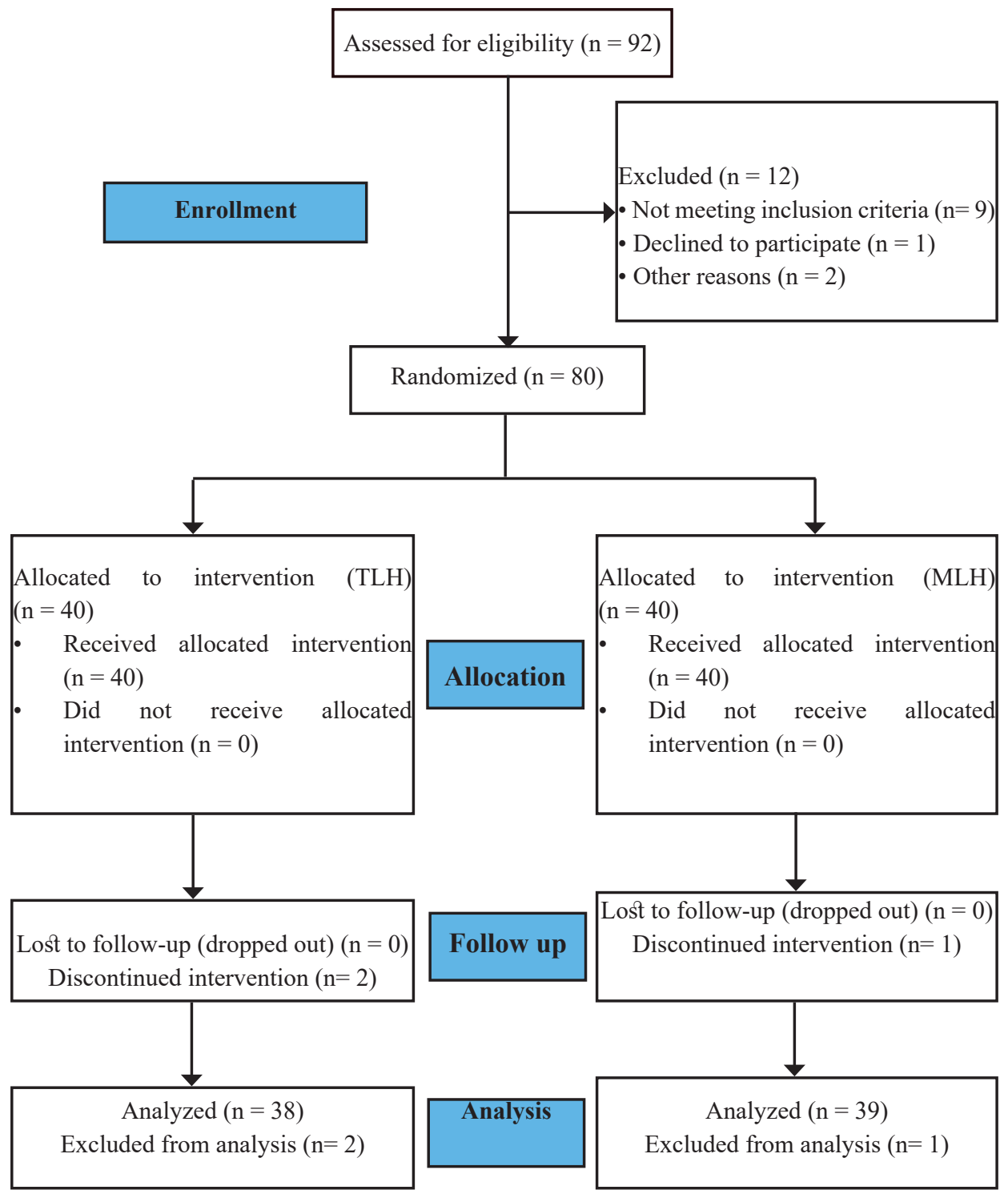

Fig. 1: Study flow diagram 
Table 1: Demographic characteristics of the study groups

\begin{tabular}{|c|c|c|c|}
\hline & TLH $(n=40)$ & $\operatorname{MLH}(n=40)$ & $P$ value \\
\hline \multicolumn{4}{|l|}{ Age (years) ${ }^{*}$} \\
\hline Mean \pm SD & $47.45 \pm 3.76$ & $48.03 \pm 3.21$ & 0.712 \\
\hline Median (min-max) & $47(4055)$ & $49(4255)$ & \\
\hline \multicolumn{4}{|l|}{$\mathrm{BMI}^{*}$} \\
\hline Mean \pm SD & $32.42 \pm 3.58$ & $30.33 \pm 4.67$ & 0.010 \\
\hline Median (min-max) & 31.99 (26.79 42.17) & 29.99 (24.98 49.05) & \\
\hline Parity $^{\dagger}$ & $3(0-6)$ & $4(1-6)$ & 0.287 \\
\hline Menopausal women & $14(35.0 \%)$ & $8(20.0 \%)$ & 0.133 \\
\hline Previous pelvic surgery ${ }^{\dagger}$ & $10(25.0 \%)$ & $9(22.5 \%)$ & 0.793 \\
\hline \multicolumn{4}{|l|}{ Uterine disorder ${ }^{\star}$} \\
\hline Fibroid & $13(32.5 \%)$ & $13(32.5 \%)$ & \\
\hline Adenomyosis & $13(32.5 \%)$ & $14(35.0 \%)$ & 0.964 \\
\hline Endometrial hyperplasia & $14(35.0 \%)$ & $13(32.5 \%)$ & \\
\hline
\end{tabular}

* Expressed as mean $\pm \mathrm{SD}$ and median (min-max) and P value was calculated by the Mann-Whitney U-test.

$\dagger$ Expressed as median and rang and $P$ value was calculated by the Mann-Whitney U-test.

\$ Expressed as frequency and percentage and $\mathrm{P}$ value was calculated by the Chi-square test.

BMI, body mass index; HCT, hematocrit; Hb, hemoglobin; max, maximum; min, minimum; MLH, mini-laparotomy hysterectomy; SD, standard deviation; TLH, total laparoscopic hysterectomy.

Table 2: Operative data among cases continued the intervention in both groups

\begin{tabular}{|c|c|c|c|}
\hline & TLH $(\mathrm{n}=38)$ & MLH $(n=39)$ & Pvalue \\
\hline \multicolumn{4}{|l|}{ Blood loss $(\mathrm{ml})^{*}$} \\
\hline Mean \pm SD & $92.11 \pm 26.40$ & $52.31 \pm 20.19$ & \multirow[t]{2}{*}{$<0.001$} \\
\hline Median (min-max) & $97.5(50-140)$ & $50(30-120)$ & \\
\hline \multicolumn{4}{|c|}{ Operative time (minutes) ${ }^{*}$} \\
\hline Mean \pm SD & $93.68 \pm 15.58$ & $65.26 \pm 8.35$ & \multirow[t]{2}{*}{$<0.001$} \\
\hline Median (min-max) & $90(70-125)$ & $70(50-75)$ & \\
\hline \multicolumn{4}{|l|}{ Uterine weight $(\mathrm{gm})^{*}$} \\
\hline Mean $\pm \mathrm{SD}$ & $224.87 \pm 51.55$ & $210.77 \pm 39.81$ & \multirow[t]{2}{*}{0.259} \\
\hline Median (min-max) & $230(130-350)$ & $210(130-280)$ & \\
\hline Adhesiolysis $^{\dagger}$ & $7(18.4 \%)$ & $6(15.4 \%)$ & 0.722 \\
\hline \multicolumn{4}{|c|}{ Salpingo-oophorectomy ${ }^{\dagger}$} \\
\hline Unilateral & $3(7.9 \%)$ & $2(5.1 \%)$ & \multirow[t]{2}{*}{0.622} \\
\hline Bilateral & $35(92.1 \%)$ & $37(94.9 \%)$ & \\
\hline
\end{tabular}

* Expressed as mean \pm SD and median (min-max) and P value was calculated by the Mann-Whitney U-test.

$\dagger$ Expressed as frequency and percentage and $P$ value was calculated by the Chi-square test.

max, maximum; min, minimum; MLH, mini-laparotomy hysterectomy; SD, standard deviation; TLH, total laparoscopic hysterectomy. 
Table 3: Change in $\mathrm{Hb}$ level and Hct value among cases continued the intervention in both groups

\begin{tabular}{|c|c|c|c|}
\hline & TLH $(\mathrm{n}=38)$ & MLH $(n=39)$ & Pvalue \\
\hline \multicolumn{4}{|c|}{ Preoperative $\mathrm{Hb}$ level (gm/dl) } \\
\hline Mean \pm SD & $12.03 \pm 1.26$ & $11.81 \pm 1.04$ & 0.491 \\
\hline Median (min-max) & $12.1(10.014 .4)$ & $11.8(10.013 .6)$ & \\
\hline \multicolumn{4}{|c|}{ Postoperative Hb level (gm/dl) } \\
\hline Mean \pm SD & $11.17 \pm 1.23$ & $11.19 \pm 1.00$ & 0.992 \\
\hline Median (min-max) & $11.2(9.0-13.5)$ & $11.1(9.512 .9)$ & \\
\hline \multicolumn{4}{|l|}{ Drop in $\mathrm{Hb}$ level (\%) } \\
\hline Mean \pm SD & $7.18 \pm 2.94$ & $5.32 \pm 1.54$ & 0.001 \\
\hline Median (min-max) & $7.11(1.52-17.43)$ & $5.56(1.53-7.69)$ & \\
\hline \multicolumn{4}{|c|}{ Preoperative Hct value (\%) } \\
\hline Mean \pm SD & $38.40 \pm 3.53$ & $37.09 \pm 2.87$ & 0.077 \\
\hline Median (min-max) & $39(3045)$ & $37(3243.5)$ & \\
\hline \multicolumn{4}{|c|}{ Postoperative Hct value (\%) } \\
\hline Mean \pm SD & $34.35 \pm 3.44$ & $33.02 \pm 2.80$ & 0.063 \\
\hline Median (min-max) & $34.75(2843)$ & $33(27-40)$ & \\
\hline \multicolumn{4}{|c|}{ Drop in Hct value (\%) } \\
\hline Mean \pm SD & $10.52 \pm 3.86$ & $10.89 \pm 4.90$ & 0.572 \\
\hline Median (min-max) & $10.00(4.4419 .75)$ & $10.26(0.0029 .89)$ & \\
\hline
\end{tabular}

Data are expressed as mean $\pm \mathrm{SD}$ and median (min-max) and $P$ value was calculated by the Mann-Whitney U-test.

Hct, hematocrit; Hb, hemoglobin; max, maximum; min, minimum; MLH, mini-laparotomy hysterectomy; SD, standard deviation; TLH, total laparoscopic hysterectomy.

Table 4: Complications among the studied groups

\begin{tabular}{|c|c|c|c|}
\hline & TLH $(n=40)$ & MLH $(n=40)$ & Pvalue \\
\hline \multicolumn{4}{|l|}{ Intraoperative complications } \\
\hline Bleeding requiring blood transfusion & $1(2.5 \%)$ & 0 & 1.000 \\
\hline Visceral injury & $1(2.5 \%)$ & 0 & 1.000 \\
\hline Conversion & $2(5 \%)$ & $1(2.5 \%)$ & 1.000 \\
\hline \multicolumn{4}{|l|}{ Postoperative complications } \\
\hline Paralytic ileus & $1(2.5 \%)$ & 0 & 1.000 \\
\hline Wound infections & 0 & $2(5 \%)$ & 0.494 \\
\hline
\end{tabular}

Data are expressed as frequency and percentage and P value was calculated by the Fischer's exact.

max, maximum; min, minimum; MLH, mini-laparotomy hysterectomy; SD, standard deviation; TLH, total laparoscopic hysterectomy.

\section{DISCUSSION}

There is a gap in the literature as there are no RCTs comparing the perioperative outcomes with respect to estimated blood loss and related early postoperative complications between MLH and TLH in patients with benign uterine lesions. The only reported two studies comparing MLH with TLH have a retrospective design that may lead to a selection bias and inconclusive results ${ }^{[14,15]}$. Our RCT demonstrated that MLH has significantly lower operative time and lower amount of estimated blood loss. The percentage of postoperative drop in $\mathrm{Hb}$ level was significantly higher in the TLH group than in the MLH arm and this finding probably reflects a status of more acute blood loss in favor of TLH procedure. Both techniques were comparable in terms of intraoperative complications rate, conversion rate, changes in the Hct value, and related early postoperative complications rate.
The current evidence is that $\mathrm{VH}$ is the first preferred approach for benign disease and laparoscopic hysterectomy acts as a preferable alternative approach to open abdominal hysterectomy when $\mathrm{VH}$ is not indicated or feasible ${ }^{[6-8]}$. Despite VH is the first preferred approach, there are relative contraindications for $\mathrm{VH}$ such as previous $\mathrm{CS}$, nulliparity or no prior vaginal delivery, large uterus, lack of uterine descent and planned oophorectomy ${ }^{[9]}$. Laparoscopic hysterectomy has several advantages compared with the conventional abdominal hysterectomy, including reduction in postoperative pain, less blood loss, shorter hospital stay and faster recovery; however, its drawbacks are longer operative time, expensive equipment, required special training and a longer learning curve ${ }^{[7]}$.

Perron-Burdick et $a l^{[14]}$ conducted a retrospective study comparing laparoscopic hysterectomy with MLH. The laparoscopic technique demonstrated a significantly 
lower amount of blood loss. This finding contradicts that reported in our prospective trial. This could be explained by the retrospective study design nature reported by that analysis coupled with the difference in the surgical technique. However, the investigators stated that the MLH group experienced a significantly shorter procedure time with no significant difference between both groups with respect to patient morbidity; including intraoperative and postoperative complications and these data go hand by hand with that demonstrated in our trial.

Our results confirm that reported in another recently published retrospective study carried on 100 patients comparing TLH with MLH $^{[15]}$. The investigators found significantly shorter operative time with MLH without a difference in terms of blood loss and complications when compared with TLH. Despite there were more intraoperative complications in the TLH arm ( 2 bladder injuries and 1 major bleeding that required blood transfusion), it did not reach a statistical significance. In our study, there were 1 case with bladder injury and another case with intraoperative bleeding requiring blood transfusion during TLH procedure with no intraoperative complications during MLH. These findings may suggest a wide safety surgical margin during MLH procedure compared with TLH technique. Furthermore, and similar to our findings, the hospital stay days were significantly longer in the MLH group but this may not had a clinical significance.

Several studies compared LAVH with MLH to evaluate the complications rates, operative times, and duration of hospitalization of both surgical procedures ${ }^{[20-23]}$. Muzii et al. conducted a prospective randomized multicenteric study on 81 patients to LAVH with MLH. They observed significantly longer operative time with LAVH, but without statistically significant differences in postoperative $\mathrm{Hb}$ drop $^{[21]}$. These data were confirmed in another prospective study comparing LAVH and MLH where the authors found a significantly shorter operative time with MLH, but without significant difference in blood loss, intraoperative and postoperative complications ${ }^{[22]}$.

In our locality, TLH is potentially more expensive than MLH (approximately $\$ 1200$ vs $\$ 300$ ) including hospitalization, instrumentations and postoperative costs. Actually, we did not measure operative costs and thus it is not clear if the difference translates to overall decreased hospital utilization or an improved cost profile. Although the cost analysis was not directly available for our study participants and was not decided to be one of our outcome measures, an economic difference analysis of hysterectomy by different surgical approaches has not been previously published in our region. In low-income countries, like Egypt, we do not have an integrated health care delivery system that provides a substantial medical care for insured adults. Within this context, the classic simple traditional surgical approaches which provide more or less the same satisfactory outcome measures and with less expenses have a higher privilege of choice.
The main strength point in our study comes from that it was the first RCT with relatively good number of cases that compared TLH with MLH. Another positive point is that patients with relative contraindications for laparoscopy, such as previous pelvic surgeries were not excluded from the study. Furthermore, all operations were performed by the same surgeon thus reducing variation in the surgical skills and the subsequent perioperative outcomes.

One limitation related to our study is the method of randomization determined by the patient's identification number. Although the ideal method is that obtained by the computer allocation, our study was assumed to be of adequate power. The cases were not only properly selected but also strictly accounted for the report (including 'dropouts'). Furthermore, the follow-up was completed for all participants. Further well-controlled prospective studies are requested to confirm or refute our findings.

\section{CONCLUSION}

MLH, compared with TLH, is a good alternative minimally invasive surgical technique for women with benign uterine disorders. The procedure offers favorable outcomes in terms of less operative time, less intraoperative bleeding, and short learning curve. Its safety, simplicity and lower cost probably give the technique a higher privilege of choice in communities with low resource settings.

\section{CONFLICT OF INTERESTS}

There are no conflicts of interest.

\section{REFERENCES}

1. Lefebvre G, Allaire C, Jeffrey J, Vilos G. No. 109-Hysterectomy. J Obstet Gynaecol Can. 2018;40(7):e567-e79.

2. Cohen SL, Vitonis AF, Einarsson JI. Updated hysterectomy surveillance and factors associated with minimally invasive hysterectomy. Jsls. 2014;18(3).

3. David-Montefiore E, Rouzier R, Chapron C, Darai E. Surgical routes and complications of hysterectomy for benign disorders: a prospective observational study in French university hospitals. Hum Reprod. 2007;22(1):260-5.

4. Stang A, Merrill RM, Kuss O. Nationwide rates of conversion from laparoscopic or vaginal hysterectomy to open abdominal hysterectomy in Germany. Eur $\mathrm{J}$ Epidemiol. 2011;26(2):125-33.

5. Wilson LF, Pandeya N, Mishra GD. Hysterectomy trends in Australia, 2000-2001 to 2013-2014: joinpoint regression analysis. Acta Obstet Gynecol Scand. 2017;96(10):1170-9. 
6. AAGL position statement: route of hysterectomy to treat benign uterine disease. J Minim Invasive Gynecol. 2011;18(1):1-3.

7. Aarts JW, Nieboer TE, Johnson N, Tavender E, Garry R, Mol BW, Kluivers KB. Surgical approach to hysterectomy for benign gynaecological disease. Cochrane Database Syst Rev. 2015(8):Cd003677.

8. Committee Opinion No 701: Choosing the Route of Hysterectomy for Benign Disease. Obstet Gynecol. 2017;129(6):e155-e9.

9. Veronikis DK. Vaginal Hysterectomy: The Present Past. Mo Med. 2015;112(6):439-42.

10. Semm K. Operationslehre für endoskopische Abdominal-Chirurgie. New York, Schattauer: Stuttgart; 1984. 485 p.

11. Reich H, deCaprio J, Mcglynn F. Laparoscopic hysterectomy. J Gynecol Surg. 1989;5(2):213-6.

12. Newcomb WL, Hope WW, Schmelzer TM, Heath JJ, Norton HJ, Lincourt AE, Heniford BT, Iannitti DA. Comparison of blood vessel sealing among new electrosurgical and ultrasonic devices. Surg Endosc. 2009;23(1):90-6.

13. Pelosi MA, 2nd and Pelosi MA, 3rd. Pelosi minilaparotomy hysterectomy: a non-endoscopic minimally invasive alternative to laparoscopy and laparotomy. Surg Technol Int. 2004;13:157-67.

14. Perron-Burdick M, Calhoun A, Idowu D, Pressman A, Zaritsky E. Minilaparotomy vs. laparoscopic hysterectomy: comparison of length of hospital stay. J Minim Invasive Gynecol. 2014;21(4):619-23.
15. Sirisabya N, Manchana T. Minilaparotomy vs laparoscopic hysterectomy for benign gynaecological diseases. J Obstet Gynaecol. 2014;34(1):65-9.

16. Wright KN, Jonsdottir GM, Jorgensen S, Shah N, Einarsson JI.Costs and Outcomes of Abdominal, Vaginal, Laparoscopic and Robotic Hysterectomies. JSLS 2012; 16:519-524

17. Einarsson JI, Suzuki Y. Total laparoscopic hysterectomy: 10 steps toward a successful procedure. Rev Obstet Gynecol. 2009;2(1):57-64.

18. Fanfani F, Fagotti A, Longo R, Marana E, Mancuso S, Scambia G. Minilaparotomy in the management of benign gynecologic disease. Eur J Obstet Gynecol Reprod Biol. 2005;119(2):232-6.

19. Schorn MN. Measurement of blood loss: review of the literature. J Midwifery Womens Health. 2010;55(1):20-7.

20. Benedetti-Panici P, Maneschi F, Cutillo G, Scambia G, Congiu M, Mancuso S. Surgery by minilaparotomy in benign gynecologic disease. Obstet Gynecol. 1996;87(3):456-9.

21. Muzii L, Basile S, Zupi E, Marconi D, Zullo MA, Manci N, Bellati F, Angioli R, Benedetti Panici P. Laparoscopic-assisted vaginal hysterectomy versus minilaparotomy hysterectomy: a prospective, randomized, multicenter study. J Minim Invasive Gynecol. 2007;14(5):610-5.

22. Agarwal A, Shetty J, Pandey D. Feasibility and Compatibility of Minilaparotomy Hysterectomy in a Low-Resource Setting. Obstetrics and Gynecology International 2018;2018:8354272. 